This article has been accepted for publication in Monthly Notices of the Royal Astronomical Society. Letters (C: 2020 The Authors. Published by Oxford University Press on behalf of the Royal Astronomical Society. All rights reserved. 


\title{
Discovering the most elusive radio relic in the sky: diffuse shock acceleration caught in the act?
}

\author{
Nicola T. Locatelli, ${ }^{1,2 \star}$ Kamlesh Rajpurohit $^{\oplus},{ }^{1}$ Franco Vazza ${ }^{\oplus}, 1,2,3$ \\ Fabio Gastaldello ${ }^{\odot},{ }^{4}$ Daniele Dallacasa, ${ }^{1,2}$ Annalisa Bonafede, ${ }^{1,2}$ \\ Mariachiara Rossetti, ${ }^{4}$ Chiara Stuardi, ${ }^{1,2}$ Etienne Bonassieux, ${ }^{1}$ \\ Gianfranco Brunetti, ${ }^{2}$ Marcus Brüggen ${ }^{4}$ and Timothy Shimwell ${ }^{5,6}$ \\ ${ }^{1}$ Dipartimento di Fisica, Astronomia DIFA, Universitá di Bologna, via Gobetti 92, I-40126 Bologna, Italy \\ ${ }^{2}$ INAF - Istituto di Radioastronomia, via Gobetti 92, I-40129 Bologna, Italy \\ ${ }^{3}$ Hamburger Sternwarte, University of Hamburg, Gojenbergsweg 112, D-21029 Hamburg, Germany \\ ${ }^{4}$ INAF - IASF Milano, Via Bassini 15, I-20133 Milano, Italy \\ ${ }^{5}$ Leiden Observatory, Leiden University, PO Box 9513, NL-2300 RA Leiden, the Netherlands \\ ${ }^{6}$ ASTRON, the Netherlands Institute for Radio Astronomy, Postbus 2, NL-7990 AA Dwingeloo, the Netherlands
}

Accepted 2020 April 20. Received 2020 April 20; in original form 2020 March 19

\begin{abstract}
The origin of radio relics is usually explained via diffusive shock acceleration (DSA) or reacceleration of electrons at/from merger shocks in galaxy clusters. The case of acceleration is challenged by the low predicted efficiency of low Mach number merger shocks, unable to explain the power observed in most radio relics. In this letter, we present the discovery of a new giant radio relic around the galaxy cluster Abell $2249(z=0.0838)$ using Low-Frequency Array (LOFAR). It is special since it has the lowest surface brightness of all known radio relics. We study its radio and X-ray properties combining LOFAR data with UGMRT, JVLA, and XMM. This object has a total power of $L_{1.4 \mathrm{GHz}}=4.1 \pm 0.8 \times 10^{23} \mathrm{~W} \mathrm{~Hz}^{-1}$ and integrated spectral index $\alpha=1.15 \pm 0.23$. We infer for this radio relic a lower bound on the magnetization of $B \geq 0.4 \mu \mathrm{G}$, a shock Mach number of $\mathcal{M} \approx 3.79$, and a low acceleration efficiency consistent with DSA. This result suggests that a missing population of relics may become visible, thanks to the unprecedented sensitivity of the new generation of radio telescopes.
\end{abstract}

Key words: acceleration of particles - magnetic fields-galaxies: clusters: general.

\section{INTRODUCTION}

Radio relics are elongated, arc-shaped diffuse synchrotron sources extended over $\sim \mathrm{Mpc}$, usually found at the periphery of clusters of galaxies with ongoing mergers, showing with steep spectrum $\left(\alpha>1\right.$, where the flux density $S_{v}$ is defined as $S_{v} \propto v^{-\alpha}$ and $\alpha$ is the spectral index) steepening towards the cluster centre (e.g. van Weeren et al. 2019, for a review). Radio relics are strongly polarized at high frequencies, with a polarization fraction that can go up to $20-30$ per cent at $1.4 \mathrm{GHz}$ and $\sim 70$ per cent at $5 \mathrm{GHz}$ (van Weeren et al. 2010; Kierdorf et al. 2017; Loi et al. 2017). Several radio relics have also been found to trace the position of shock waves, detected as discontinuities in the X-ray brightness profiles of the intracluster medium (ICM; Akamatsu \& Kawahara 2013; Botteon, Gastaldello \& Brunetti 2018). Merger shock waves are believed to be generated when clusters of galaxies collide, and

^E-mail: nicola.locatelli2@unibo.it then propagate along the direction of the merger. Shocks are more easily seen edge-on as projection boosts their surface brightness, and the same observational bias should also apply to radio relics. The kinetic energy dissipated at shocks should be related to the powering of the radio emission, via diffusive shock acceleration (DSA; Bell 1978; Jones \& Ellison 1991), as originally proposed by Ensslin et al. (1998). However, the Mach numbers that are independently inferred from discontinuities observed in X-rays are generally too weak $(\mathcal{M} \sim 2)$ to account for the required electron acceleration efficiency by DSA in relics (e.g. Botteon et al. 2020, hereafter $\mathrm{B}+20$ ). Moreover, shock waves in the ICM should also accelerate protons that would create $\gamma$-ray emission in the collision with the thermal protons of the ICM. These $\gamma$-rays have not been detected (Ackermann et al. 2016), which translates into limits on the maximum acceleration efficiency of protons in structure formation shocks ( $<10^{-3}$; Vazza et al. 2016). This conundrum can be bypassed when invoking a pre-existing population of mildly non-thermal electrons that get re-accelerated by the shocks (Markevitch et al. 2005; Pinzke, Oh \& Pfrommer 2013; Kang \& Ryu 2015). In a few 
cases, active galactic nuclei could have supplied the relativistic electrons in the upstream region of the shock that creates the relic (Bonafede et al. 2014; van Weeren et al. 2017; Stuardi et al. 2019). Both acceleration and re-acceleration processes operate in the ICM and should contribute to the population of radio relics. We note that we adopted a flat $\Lambda$ cold dark matter cosmology with $H_{0}=69.6 \mathrm{~km} \mathrm{~s}^{-1} \mathrm{Mpc}^{-1}$ and $\Omega_{\mathrm{M}}=0.286$ throughout the paper.

\subsection{General properties of Abell 2249}

In this work, we present the discovery of a giant radio relic found at the periphery of the galaxy cluster Abell 2249 (hereafter A2249; RA 257.44080, Dec. 34.45566). Its galaxies and ICM features have been studied in detail at various wavelengths by a number of authors: the cluster mean redshift is $z=0.0838$ (Bulbul et al. 2016; Lopes et al. 2018; Laganá, Durret \& Lopes 2019); the velocity dispersion of its constituent galaxies is between $\sigma_{\text {vel }}=$ $894 \pm 50$ (Lopes et al. 2018) and $976 \pm 38 \mathrm{~km} \mathrm{~s}^{-1}$ (Oh et al. 2018). Laganá et al. (2019) provided detailed XMM-Newton maps of temperature (peaking in the 4-7 keV energy band), pseudopressure, pseudo-entropy, and metallicity in the central region, within the first $\sim 400 \mathrm{kpc}$ from the cluster centre. They classified A2249 as a non-cool-core disturbed cluster, although Oh et al. (2018) and Lopes et al. (2018) did not find evidence for merging from the spectroscopic redshift distribution of cluster members. However, a Dressler and Shectman three-dimensional test of the galaxy redshifts suggests that the cluster is disturbed (Lopes et al. 2018). The radius of the cluster is $R_{500}=1.56 \pm 0.06,1.1_{-0.1}^{+0.3} \mathrm{Mpc}$ depending on the mass estimate, which is debated between the values $M_{500}=11.7 \pm 1.4,4.0_{-1.1}^{+5.2}$, and $3.73_{-0.19}^{+0.18}$, in units of $10^{14} \mathrm{M}_{\odot}$, derived from radial velocity distribution, Chandra and Planck data, respectively (Planck Collaboration XXVII 2016; Zhu et al. 2016; Lopes et al. 2018). At larger radii, $R_{200}=2.2 \pm 0.1 \mathrm{Mpc}$ and $M_{200}=$ $12.7 \pm 1.5 \times 10^{14} \mathrm{M}_{\odot}$ (Lopes et al. 2018; Oh et al. 2018).

\section{OBSERVATION AND DATA REDUCTION}

\subsection{Radio observations}

The low-frequency observations of the A2249 field were carried out with the Low-Frequency Array (LOFAR). The LOFAR HBA (120$168 \mathrm{MHz}$ ) observation was carried out during cycle 9 (proposal ID: LC9_020). The centre of the pointing was not at the cluster centre, but at coordinates 17:01:13 + 33:20:15 (RA, Dec.), at a distance of $2.1 \mathrm{deg}$. The on-source time is $8 \mathrm{~h}$ with two scans of $10 \mathrm{~min}$ each on the flux calibrator 3C 295. A first calibration and imaging run was performed using the LOFAR data reduction pipeline $(\mathrm{v} 2.2)^{1}$ involving both direction-independent (de Gasperin et al. 2019) and direction-dependent calibration of the data (Shimwell et al. 2017). Exploiting the sky models derived from the pipeline, we subtracted from the UV data all sources outside a $1.9^{\circ} \times 1.9^{\circ}$ region centred on the relic. This was done using the Python Blob Detector and Source Finder (Mohan \& Rafferty 2015). The resulting data were then self-calibrated through nine iteration steps and then imaged using WSCLEAN v2.4 (Offringa et al. 2014).

We produced images at 6 (Fig. 1, left-hand panel) and 20 arcsec resolution using a Briggs weighting scheme with robust -0.5 . The image at higher (lower) resolution has an rms noise floor of $230(350) \mu J y$ per beam. We determined and applied a correction

\footnotetext{
${ }^{1}$ https://github.com/mhardcastle/ddf-pipeline
}

factor (see also Hardcastle et al. 2016; van Weeren et al. 2016) to match the LOFAR HBA flux densities of point-like sources with the ones derived from the TIFR GMRT Sky Survey (Intema et al. 2017). We assume flux uncertainties of 20 per cent, similar to the LOFAR Two-meter Sky Survey images (Shimwell et al. 2019).

We also observed the cluster with the uGMRT, in Band 4 covering a frequency range of 550-950 MHz (proposal DDT-C100). The data were flagged and calibrated using Common Astronomy Software Applications (CASA). We then ran several rounds of directiondependent self-calibration using the LOFAR DDF-pipel ine (see above). The image reaches a noise level of $16 \mu \mathrm{Jy}$ perbeam at $700 \mathrm{MHz}$.

We have also analysed two short snapshot observations at $1.46 \mathrm{GHz}$ from the Very Large Array (VLA) archive. About 8 min (four 2-min scans well spaced in time) and 25 min (single scan) in C and D configurations were available (project codes AS220 and AG294, respectively). We obtained a combined image of the intersecting part of the bands after standard calibration of the two individual data sets. The pointing was set on the brightest central galaxy (BCG), which is about 15 arcmin off the relic position. This highly affected the local sensitivity. The combined $\mathrm{C}+\mathrm{D}$ image (not shown) allowed a resolution of about 30 arcsec and presents a number of separate patches of diffuse emission with peaks just above the local $3 \sigma$ in the region of the relic, with roughly the same morphology of the uGMRT image.

\subsection{X-ray: $X M M$ observation}

A2249 (also known under the name PSZ2 G057.61+34.93) has been observed as part of the XMM Heritage Cluster Project ${ }^{2}$ (PI: Arnaud \& Ettori, in preparation), a large and unbiased sample of 118 clusters, detected with a high signal-to-noise ratio in the second Planck SZ catalogue. We reduced the data with SAS v 16.1. The observation with OBSID 0827010501 has a total clean exposure time of $20.4 \mathrm{ks}$ with MOS1, 20.7 with MOS2, and 16.1 with pn after filtering for soft proton flares (81 per cent of the total time for MOS and 93 per cent for the pn). We estimated the amount of residual soft protons following the procedure described in Cova et al. (2019) and found it to be negligible. For a full description of data reduction, image production, and spectral extraction, we refer to Ghirardini et al. (2019). In the right-hand panel of Fig. 1, we show the $X M M$ image in the $0.7-1.2 \mathrm{keV}$ band with the overlay of the radio contours at $150 \mathrm{MHz}$ with 6 arcsec resolution and the regions used for the spectral analysis. Given that the emission of the cluster is filling the entire field of view of XMM for the estimate of the sky background components in a similar way to Snowden et al. (2008), we used a spectrum from the ROSAT All-Sky Survey extracted from an anulus between 0.5 and $1 \mathrm{deg}$ from the source. We fixed the Galactic $N_{\mathrm{H}}$ to $2.38 \times 10^{20} \mathrm{~cm}^{-2}$ at HI LAB value (Kalberla et al. 2005), given the negligible difference with the value $\left(2.5 \times 10^{22} \mathrm{~cm}^{-2}\right)$ that estimates the possible contribution of molecular hydrogen (Willingale et al. 2013).

\section{RESULTS}

\subsection{Morphology}

The extended diffuse emission at $144 \mathrm{MHz}$ (Fig. 1) is arc-shaped and oriented perpendicular to the radial direction from the cluster

\footnotetext{
${ }^{2}$ http://xmm-heritage.oas.inaf.it
} 

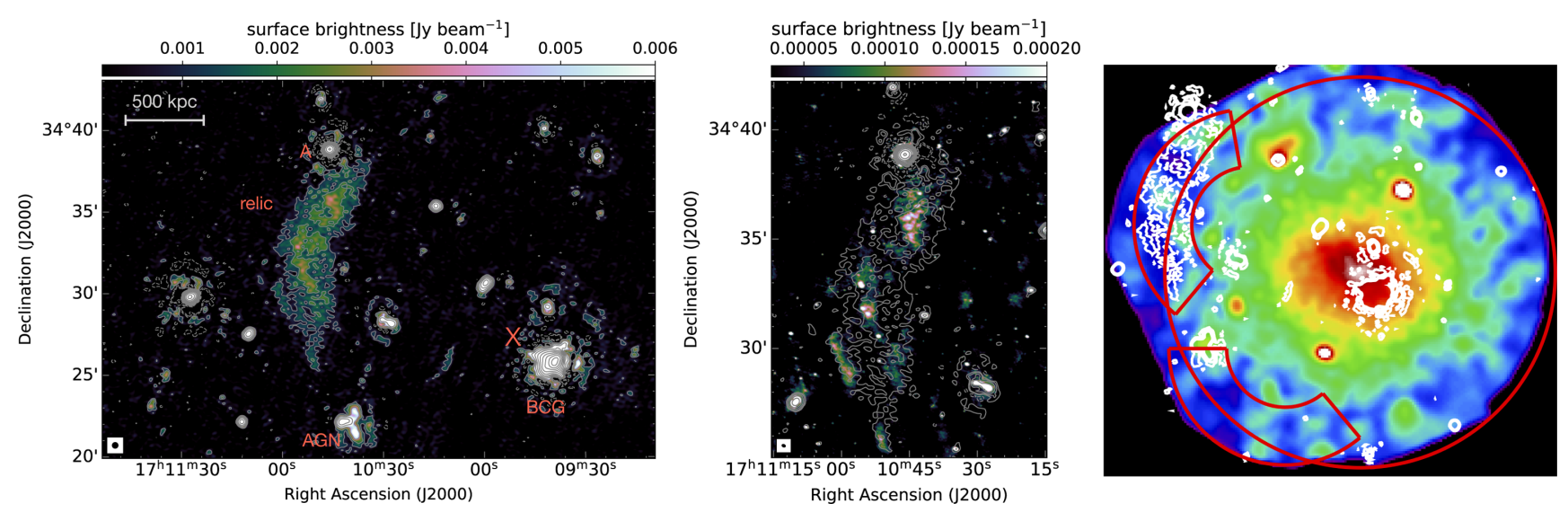

Figure 1. Left: LOFAR low-resolution (20 arcsec) image of Abell 2249, showing a spectacular large-scale radio relic. The red cross marks the cluster centre. Contour levels are drawn at $[1,2,4,8, \ldots] \times 3 \sigma_{\text {rms }}$ and are from the LOFAR image. Negative $-3 \sigma_{\text {rms }}$ contours are shown with dotted lines. Centre: Upgraded Giant Meter Radio Telescope (uGMRT) high-resolution $(8 \operatorname{arcsec} \times 6 \operatorname{arcsec})$ image of the relic, overlaid with LOFAR contours, revealing filamentary substructures. Right: Background-subtracted, exposure-corrected, and adaptively smoothed XMM image in the 0.7-1.2 keV band of A2249. The 144 MHz contours at $3 \sigma, 6 \sigma$, and $10 \sigma$ of the low-resolution LOFAR radio emission are overlaid in white. A circle of radius 14 arcmin is drawn to guide the eye for the two sectors used in the spectral analysis described in the text: one encompassing the relic radio emission and one test region of the same extension at the same radial distance from the cluster centre.

centre, in the north-east-east sector of A2249, spanning an angular radial range $[11.0 ; 17.0]$ arcmin from the cluster centre. The relic width is maximal at its mean azimuthal direction and is minimal at the azimuthal ends of the diffuse emission, giving the radio relic a shape very similar to a crescent moon or the popular Italian sweet bun named 'cornetto'. The brightest part of the relic at $144 \mathrm{MHz}$ is found at an angular radial distance of $\simeq 14.7 \mathrm{arcmin}$, which is a linear distance of $1.40 \mathrm{Mpc}$ at the redshift of A2249. The relic's largest angular scale (LAS) is $\simeq 13.2$ arcmin, corresponding to a physical size of $1.3 \mathrm{Mpc}$ at the redshift of the cluster. The northern end of the diffuse emission coincides with a bright unresolved radio source (A, Fig. 1, left-hand panel), of 400 mJy per beam at $144 \mathrm{MHz}$. Visible in the south-west direction is the BCG of A2249. Deconvolution artefacts remained around the bright sources A and BCG. The relic also shows elongated patches of emission of a few arcminutes, in analogy with the filamentary structures described in other radio relics (Owen et al. 2014; Pearce et al. 2017; Rajpurohit et al. 2018), whose origin is still unclear. The image at $700 \mathrm{MHz}$ also shows diffuse emission at the relic position above $3 \sigma$, with a similar morphology to that at lower frequency, as well as a large density of point sources (Fig. 1, central panel).

\subsection{Radio spectrum and luminosity}

The flux density and luminosity of the Cornetto relic at $144 \mathrm{MHz}$ are $F_{144 \mathrm{MHz}}=370 \pm 70 \mathrm{mJy}$ and $L_{144 \mathrm{MHz}}=5.9 \pm 1.2 \times$ $10^{24} \mathrm{~W} \mathrm{~Hz}^{-1}$, respectively. The integrated spectral index, calculated from the ratio of the total flux densities at 144 and $700 \mathrm{MHz}$ in the relic region (determined at $144 \mathrm{MHz}$ ), is $\alpha=1.15 \pm 0.23$. The observed quantities are summarized in Table 1.

Assuming $\alpha=1.15$ to be constant, we extrapolated the luminosity at $1.4 \mathrm{GHz}$ to be $L_{1.4 \mathrm{GHz}}=4.1 \pm 0.8 \times 10^{23} \mathrm{~W} \mathrm{~Hz}^{-1}$. The Cornetto relic (red star, Fig. 2) is found to lie below the observed scaling relation between the radio power at $1.4 \mathrm{GHz}$ and the largest linear size (LLS) of a sample of known radio relics presented in Nuza et al. (2017), extracted from the NRAO VLA Sky Survey (NVSS; Condon et al. 1998). From archival VLA images, we find three different regions across the relic with matching $3 \sigma$ contours between $144 \mathrm{MHz}$ and $1.4 \mathrm{GHz}$. We computed the integrated power for these
Table 1. Properties of the Cornetto relic in Abell $2249(z=0.0838)$.

\begin{tabular}{|c|c|c|}
\hline Symbol & Value & Description \\
\hline$F_{144 \mathrm{MHz}}$ & $370 \pm 70 \mathrm{mJy}$ & Flux density at $144 \mathrm{MHz}$ \\
\hline$L_{144 \mathrm{MHz}}$ & $5.9 \pm 1.2 \times 10^{24} \mathrm{~W} \mathrm{~Hz}^{-1}$ & Luminosity at $144 \mathrm{MHz}$ \\
\hline$F_{700 \mathrm{MHz}}$ & $60 \pm 12 \mathrm{mJy}$ & Flux density at $700 \mathrm{MHz}$ \\
\hline$\alpha_{700 \mathrm{MHz}, \text { int }}^{144 \mathrm{MHz}}$ & $1.15 \pm 0.23$ & $\begin{array}{l}\text { Integrated spectral index } \\
\text { Between } 144 \text { and } 700 \mathrm{MHz}\end{array}$ \\
\hline$\Delta \Omega_{144 \mathrm{MHz}}$ & $28.46 \operatorname{arcmin}^{2}$ & $\begin{array}{c}\text { Relic solid angle at } \\
144 \mathrm{MHz}\end{array}$ \\
\hline$R_{\text {proj }}$ & $1.40 \mathrm{Mpc}$ & $\begin{array}{c}\text { Projected radial cluster } \\
\text { distance }\end{array}$ \\
\hline LAS & $13.2 \operatorname{arcmin}$ & Largest angular scale \\
\hline $\operatorname{LLS}\left(z_{\mathrm{A} 2249}\right)$ & $1.3 \mathrm{Mpc}$ & Largest linear scale \\
\hline
\end{tabular}

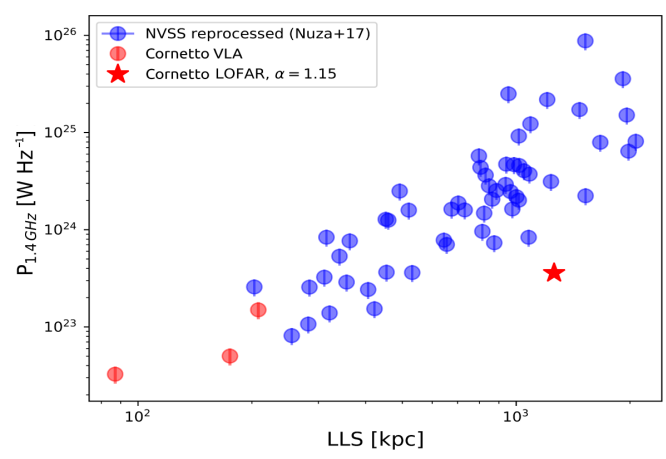

Figure 2. The luminosity at $1.4 \mathrm{GHz}$ is plotted against the LLS for the radio relics detected in the NVSS (Nuza et al. 2017). The red star shows the power of the Cornetto relic extrapolated to $1.4 \mathrm{GHz}$.

three regions and plotted them in Fig. 2 (red circles). The correlation in Fig. 2 has already been shown to be determined largely by the NVSS sensitivity (Nuza et al. 2017). The LOFAR observations presented here seem to open the window to a population of faint and diffuse relics that have not been seen to date. 


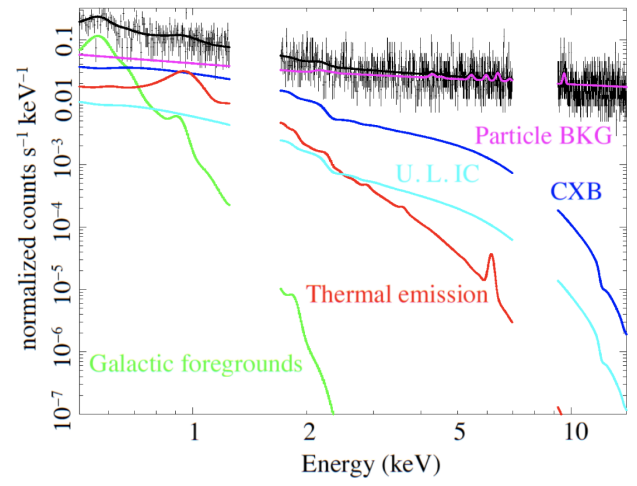

Figure 3. $X M M$ pn spectrum extracted from the region of the relic radio emission. The magenta line shows the instrumental background, the green one the galactic foregrounds, the blue one the cosmic X-ray background, the red one the ICM thermal emission, and the cyan one the 90 per cent upper limit on the IC power law.

\subsection{X-ray properties at the position of the relic}

We extracted $X M M$ MOS and pn spectra from an angular sector that covers the relic radio emission as shown in Fig. 1, right-hand panel. The region extends beyond $R_{500}$ and therefore the thermal emission is below the background. The temperature obtained is prone to large systematic errors and we therefore rely on the value obtained within the full annulus of $k T=3.0 \pm 1.3 \mathrm{keV}$ together with an electron density $n_{\mathrm{e}}=6.4 \pm 1.5 \times 10^{-4} \mathrm{~cm}^{-3}$. Assuming that temperature, we modelled the expected inverse Compton (IC) emission as a power law with fixed photon index of 2.15 as derived from the radio spectral index and extrapolated a 90 per cent upper limit of $1.0 \times 10^{-13} \mathrm{erg} \mathrm{cm}^{-2} \mathrm{~s}^{-1}$ in the $20-80 \mathrm{keV}$ range. The $\mathrm{X}$ ray spectrum in the relic region and its modelling is shown in Fig. 3. It is equivalent to the spectrum extracted from a region at the same radial distance from the cluster but avoiding the relic emission (see Fig. 1), confirming that any IC emission is clearly subdominant.

\section{MODELLING OF PHYSICAL PROPERTIES}

Based on our observations, we study the origin of the relic in A2249 and infer limits on its magnetic field.

\subsection{Diffusive shock acceleration}

Assuming DSA, the power emitted by the Cornetto relic can be related to its shock properties (e.g. Hoeft et al. 2008, hereafter HB08; B+20):

$L_{v, \text { obs }}=C \times \frac{A}{\mathrm{Mpc}^{2}} \times \frac{n_{\mathrm{e}, \mathrm{d}}}{10^{-4} \mathrm{~cm}^{-3}} \times \xi_{\mathrm{e}} \times \frac{T_{\mathrm{e}, \mathrm{d}}^{3 / 2}}{v^{\alpha / 2}} \frac{B^{1+\alpha / 2}}{B^{2}+B_{\mathrm{CMB}}^{2}(z)}$,

where $A$ is the surface area of the relic, calculated as LLS $\cdot d_{\text {thick }}$ (we assume again $d_{\text {thick }}=$ LLS), $n_{\mathrm{e}, \mathrm{d}}$ is the downstream electron density, $\xi_{\mathrm{e}}$ is the (yet unknown) fixed fraction of the kinetic energy flux $\Phi_{\mathrm{e}} / \Phi_{\mathrm{k}}$ injected at the shock front into suprathermal electrons, $T_{\mathrm{e}, \mathrm{d}}$ is the downstream electron temperature, and $B_{\mathrm{CMB}}$ is the equivalent field of the cosmic microwave background evaluated at the redshift of A2249. The normalization $C$ is equal to $6.4 \times 10^{34} \mathrm{erg} \mathrm{s}^{-1} \mathrm{~Hz}^{-1}$ when $T_{\mathrm{e}, \mathrm{d}}$ is in units of $7 \mathrm{keV} k_{\mathrm{B}}^{-1}, v$ in units of $1.4 \mathrm{GHz}$, and $B$ in $\mu \mathrm{G}$.

Considering the values in Table 1 , an integrated spectral index $\alpha=$ 1.15 (holding a Mach number $\mathcal{M}=\sqrt{(\alpha+1) /(\alpha-1)}=3.79$ ) and the quantities derived from the XMM-Newton observations $k_{\mathrm{B}} T_{\mathrm{e}} \simeq$

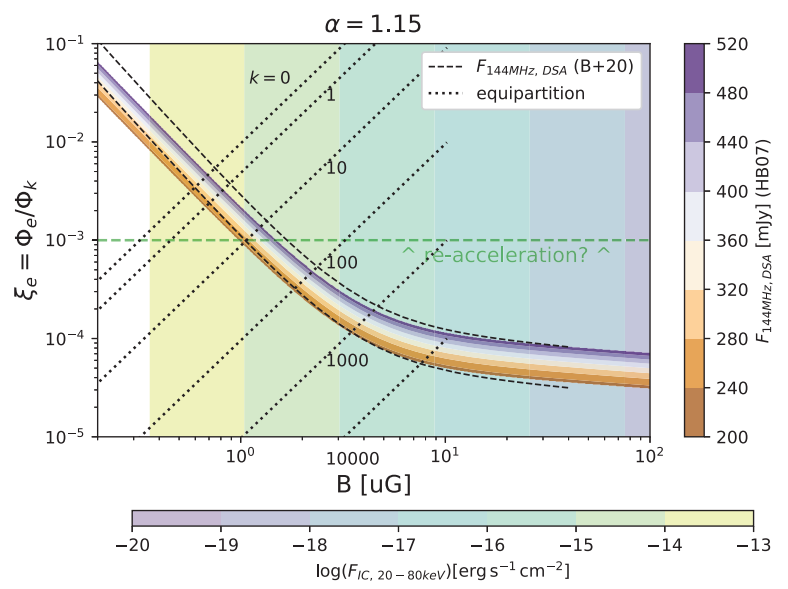

Figure 4. The $\left(B, \xi_{\mathrm{e}}\right)$ parameter space assuming $\alpha=1.15$. The curves show the points that reproduce the $F_{144 \mathrm{MHz}}$ within $2 \sigma$ uncertainty assuming DSA using HB08 (orange, white, and purple) or B+20 (dashed black) formalism. The green-violet shaded background shows the IC fluxes expected in the $20-80 \mathrm{keV}$ band. The dotted lines show the values obtained assuming equipartition for different values of $k$.

$3.0 \pm 1.3 \mathrm{keV}$ and $n_{\mathrm{e}, \mathrm{d}}=6.4 \pm 1.5 \times 10^{-4} \mathrm{~cm}^{-3}$, we can constrain the $\left(B, \xi_{\mathrm{e}}\right)$ parameter space to reproduce $F_{144 \mathrm{MHz}}$ (DSA curves in Fig. 4). For completeness, we also consider the formulation of the model as found in $\mathrm{B}+20$, which enforces the relativistic invariance in the HB08 model, which is particularly relevant for weak shocks. We obtain a magnetic field of $B=1.2 \mu \mathrm{G}$ for $\xi_{\mathrm{e}}=10^{-3}$ and $B=$ $6.0 \mu \mathrm{G}$ for $\xi_{\mathrm{e}}=10^{-4}$. The values for $\xi_{\mathrm{e}}$ agree with models for DSA from shocks with Mach numbers $\mathcal{M}=3.5-4.0$ (Kang \& Ryu 2015). Larger efficiencies are hard to reconcile with DSA and (in other objects) are used to argue for the existence of a pre-existing electron population that may have been re-accelerated by an earlier episode of shock acceleration. Re-acceleration has been invoked for most radio relics (all observed at frequencies $>600 \mathrm{MHz}$ ) for which an underlying shock wave has been detected in X-rays at their location, with the exception of the radio relic in the El Gordo galaxy cluster $(B+20)$. Instead, the efficiency required to power the Cornetto relic can be explained by DSA electrons from the thermal pool, by a few $\mu \mathrm{G}$ magnetic fields.

\subsection{Equipartition}

Synchrotron radiation provides information on both the electron's energy distribution and the magnetic field strength, $B$, in the medium. A simplistic assumption to disentangle the contribution of relativistic cosmic rays (CRs) from magnetic fields is to assume equipartition between their energy densities in the plasma $\epsilon_{\mathrm{CR}}=$ $\epsilon_{\mathrm{B}}$ (e.g. Brunetti, Setti \& Comastri 1997; Beck \& Krause 2005). In this case, the total energy density of magnetic fields and of CRs, $\epsilon_{B}+\epsilon_{C R}$, also approaches a minimum value. Classical equipartition formulae use parameters of the spectral energy distribution of electrons that is not affected by energy losses. In the case of radio relics instead, the spectrum of electrons emitting downstream results from the combination injection, transport, and energy losses. We thus derive equipartition conditions assuming that the magnetic field in radio relics gets the same energy density of particles downstream, that is

$\frac{1}{2} \rho_{\mathrm{u}} \frac{\mathrm{v}_{\mathrm{u}}^{3}}{\mathrm{v}_{\mathrm{d}}} \xi_{\mathrm{e}}(1+k)=\frac{B^{2}}{8 \pi}$, 
where $k$ is the ratio of energy budget between $\mathrm{p}$ and $\mathrm{e}, \rho$, and $\mathrm{v}$ are the gas density and shock velocity computed for the media upstream and downstream of the shock front, respectively. The jump conditions have been derived from the shock Mach number $\mathcal{M}=3.79$. With this approach, $\xi_{\mathrm{e}}$ is directly comparable with the values derived from DSA.

The results for $B, k$, and, $\xi_{\text {e }}$ are degenerate; however, the equipartition assumption alone constrains the parameter space between the curves for $k=0$ (indicating a plasma where the energy budget is only given by e) and $B \ll 10 \mu \mathrm{G}$, resulting from $\xi_{\mathrm{e}}(1+k)$ $\ll 1$. Combined with equipartition argument, the efficiency selects the value of $k$.

\subsection{IC scattering}

Based on the observed radio flux and assuming a power-law distribution of relativistic electrons, we can estimate the hard Xray emission from IC scatter from the same electron population responsible for the observed radio emission (e.g. Govoni \& Feretti 2004). Then, we can compare this to recent upper limits obtained using XMM-Newton observations in the 0.1-12 keV band. We quote the flux estimates extrapolated in the $20-80 \mathrm{keV}$ band for ease of comparison with previous estimates (e.g. Cova et al. 2019). The IC flux 90 per cent upper limit $F_{\mathrm{IC}} \leq 1 \times 10^{-13} \mathrm{erg} \mathrm{cm}^{-2} \mathrm{~s}^{-1}$ extrapolated in the $20-80 \mathrm{keV}$ band sets a lower limit on $B>$ $0.4 \mu \mathrm{G}$. A magnetic field strength of $B_{\text {low }}=0.6 \mu \mathrm{G}$ (as suggested above, assuming $\xi_{\mathrm{e}}=10^{-3}$ ) or lower would result in IC emission larger than the $F_{\mathrm{IC}} \approx 3.17 \times 10^{-14} \mathrm{erg} \mathrm{cm}^{-2} \mathrm{~s}^{-1}$ upper limit derived for A523 by Cova et al. (2019). For comparison, $\alpha=$ 1.15 and $B=6.0 \mu \mathrm{G}$ (implying $\xi_{\mathrm{e}}=10^{-4}$ for DSA) produces $F_{\mathrm{IC}} \approx 5 \times 10^{-15} \mathrm{erg} \mathrm{cm}^{-2} \mathrm{~s}^{-1}$, i.e. about one order of magnitude below present-day upper limits. The lower limit from IC combined with the limit $B \ll 10 \mu \mathrm{G}$ from energy arguments implies efficiencies $\xi_{\mathrm{e}} \in\left[5 \times 10^{-5}-10^{-2}\right]$. Larger values would violate equipartition.

\section{CONCLUSIONS}

In this letter, we presented the discovery of extended diffuse radio relic in A2249, found at low frequencies (120-168 MHz) with LOFAR. We have also observed the new relic (called Cornetto relic) at $700 \mathrm{MHz}$ with the uGMRT and found patches of emission in coincidence of the brightest parts of the relic also in VLA archival data at $1.4 \mathrm{GHz}$. The magnetic field at the relic is estimated to be $B>0.4 \mu \mathrm{G}$, depending on model assumptions and the electron acceleration efficiency $\xi_{\mathrm{e}} \leq 10^{-2}$ of the putative merger shock. The limits have been set from the absence of IC emission in the [0.1-12] keV energy band.

The Cornetto relic is among the largest relics discovered to date (13.2 arcmin, corresponding to $1.26 \mathrm{Mpc}$ ) as well as the faintest one with such extent, once extrapolated at $1.4 \mathrm{GHz}$, lying at least a factor $\sim 10$ below the observed scaling relation between the radio power at $1.4 \mathrm{GHz}$ and the LLS of radio relics.

Its low luminosity is well explained by DSA for the inferred plasma and shock parameters, unlike most other radio relics that require a higher electron acceleration efficiency and invoke past acceleration events acting on the seed electron population already present in the ICM thermal pool.

This discovery, only made possible by the unprecedented sensitivity of LOFAR to large angular scales at low frequencies, may hint to a population of low-power, faint, and diffuse radio relics, for which re-acceleration has not taken place (or not yet) or is inefficient with respect to standard DSA. This can be explored by the new generation low-frequency arrays (e.g. LOFAR, SKA-low).

\section{ACKNOWLEDGEMENTS}

We thank our anonymous reviewer for the helpful scientific feedback. NTL, KR, and FV acknowledge financial support from the ERC Starting Grant 'MAGCOW', no. 714196. AB, CS, and EB acknowledge financial support from the ERC Starting Grant 'DRANOEL', no. 714245. NTL thanks Silvia Gandolfi and Raffaele Moretti for extensive support. We thank Dan Wik for useful discussions about IC emission. MB acknowledges support from the Deutsche Forschungsgemeinschaft under Germany's Excellence Strategy - EXC 2121 'Quantum Universe' - 390833306. FG and MR acknowledge financial contribution from the agreement ASI-INAF no. 2017-14-H. Radio imaging made use of WSCLEAN v2.6 (Offringa et al. 2014) and CASA (https://casa.nrao.edu). This paper is based (in part) on data obtained with the International LOFAR Telescope (obs. ID LC9_020, PI FV) and analysed using LOFAR-IT infrastructure. LOFAR (van Haarlem et al. 2013) is the Low-Frequency Array designed and constructed by ASTRON and collectively operated by the ILT Foundation.

\section{REFERENCES}

Ackermann M. et al., 2016, ApJ, 819, 149

Akamatsu H., Kawahara H., 2013, PASJ, 65, 16

Beck R., Krause M., 2005, Astron. Nachr., 326, 414

Bell A. R., 1978, MNRAS, 182, 147

Bonafede A., Intema H. T., Brüggen M., Girardi M., Nonino M., Kantharia N., van Weeren R. J., Röttgering H. J. A., 2014, ApJ, 785, 1

Botteon A., Gastaldello F., Brunetti G., 2018, MNRAS, 476, 5591

Botteon A., Brunetti G., Ryu D., Roh S., 2020, A\&A, 634, A64 (B+20)

Brunetti G., Setti G., Comastri A., 1997, A\&A, 325, 898

Bulbul E., Markevitch M., Foster A., Miller E., Bautz M., Loewenstein M., Rand all S. W., Smith R. K., 2016, ApJ, 831, 55

Condon J. J., Cotton W. D., Greisen E. W., Yin Q. F., Perley R. A., Taylor G. B., Broderick J. J., 1998, AJ, 115, 1693

Cova F. et al., 2019, A\&A, 628, A83

de Gasperin F. et al., 2019, A\&A, 622, A5

Ensslin T. A., Biermann P. L., Klein U., Kohle S., 1998, A\&A, 332, 395

Ghirardini V. et al., 2019, A\&A, 621, A41

Govoni F., Feretti L., 2004, Int. J. Mod. Phys. D, 13, 1549

Hardcastle M. J. et al., 2016, MNRAS, 462, 1910

Hoeft M., Brüggen M., Yepes G., Gottlöber S., Schwope A., 2008, MNRAS, 391, 1511 (HB08)

Intema H. T., Jagannathan P., Mooley K. P., Frail D. A., 2017, A\&A, 598, A78

Jones F. C., Ellison D. C., 1991, Space Sci. Rev., 58, 259

Kalberla P. M. W., Burton W. B., Hartmann D., Arnal E. M., Bajaja E., Morras R., Pöppel W. G. L., 2005, A\&A, 440, 775

Kang H., Ryu D., 2015, ApJ, 809, 186

Kierdorf M., Beck R., Hoeft M., Klein U., van Weeren R. J., Forman W. R., Jones C., 2017, A\&A, 600, A18

Laganá T. F., Durret F., Lopes P. A. A., 2019, MNRAS, 484, 2807

Loi F. et al., 2017, MNRAS, 472, 3605

Lopes P. A. A., Trevisan M., Laganá T. F., Durret F., Ribeiro A. L. B., Rembold S. B., 2018, MNRAS, 478, 5473

Markevitch M., Govoni F., Brunetti G., Jerius D., 2005, ApJ, 627, 733

Mohan N., Rafferty D., 2015, Astrophysics Source Code Library, record ascl:1502.007

Nuza S. E., Gelszinnis J., Hoeft M., Yepes G., 2017, MNRAS, 470, 240

Offringa A. R. et al., 2014, MNRAS, 444, 606

Oh S. et al., 2018, ApJS, 237, 14 
Owen F. N., Rudnick L., Eilek J., Rau U., Bhatnagar S., Kogan L., 2014, ApJ, 794, 24

Pearce C. J. J. et al., 2017, ApJ, 845, 81

Pinzke A., Oh S. P., Pfrommer C., 2013, MNRAS, 435, 1061

Planck Collaboration XXVII, 2016, A\&A, 594, A27

Rajpurohit K. et al., 2018, ApJ, 852, 65

Shimwell T. W. et al., 2017, A\&A, 598, A104

Shimwell T. W. et al., 2019, A\&A, 622, A1

Snowden S. L., Mushotzky R. F., Kuntz K. D., Davis D. S., 2008, A\&A, 478,615

Stuardi C. et al., 2019, MNRAS, 489, 3905

van Haarlem M. P. et al., 2013, A\&A, 556, A2

van Weeren R. J., Röttgering H. J. A., Brüggen M., Hoeft M., 2010, Science, 330,347 van Weeren R. J. et al., 2016, ApJS, 223, 2

van Weeren R. J. et al., 2017, Nat. Astron., 1, 0005

van Weeren R. J., de Gasperin F., Akamatsu H., Brüggen M., Feretti L.,

Kang H., Stroe A., Zandanel F., 2019, Space Sci. Rev., 215, 16

Vazza F., Brüggen M., Wittor D., Gheller C., Eckert D., Stubbe M., 2016, MNRAS, 459, 70

Willingale R., Starling R. L. C., Beardmore A. P., Tanvir N. R., O’Brien P. T., 2013, MNRAS, 431, 394

Zhu Z. et al., 2016, ApJ, 816, 54

This paper has been typeset from a $\mathrm{T}_{\mathrm{E}} \mathrm{X} / \mathrm{LT}_{\mathrm{E}} \mathrm{X}$ file prepared by the author. 